\title{
Optical offset pointing of radio interferometers: applications at the Combined Array for Research in Millimeter-wave Astronomy
}

\author{
Stuartt A. Corder ${ }^{a}$, Melvyn C. H. Wright ${ }^{b}$, and John M. Carpenter ${ }^{c}$ \\ ${ }^{a}$ National Radio Astronomy Observatory, Joint Atacama Large Millimeter/Submillimeter \\ Array Observatory, 3650 Av. Apoquindo, Piso 18, Santiago, Chile; \\ ${ }^{b}$ Radio Astronomy Lab, University of California, Berkeley, 453 Campbell Hall, Berkeley, \\ California, U.S.A.; \\ ${ }^{c}$ Division of Physics, Mathematics and Astronomy, California Institute of Technology, MC \\ 249-17, Pasadena, California, U.S.A.
}

\begin{abstract}
Optical telescopes and cameras are often used to determine the initial pointing model for radio antennas. After this initial determination, the optical systems are typically not used. The Combined Array for Research in Millimeter-wave Astronomy (CARMA) has implemented optical offset pointing as a standard calibration option for science observations. We report on the proof of concept testing, the method, and the typical improvements obtained over traditional radio pointing. We conclude with a brief discussion of future directions, which may offer further improved pointing at CARMA and at other facilities that require increased pointing accuracy.
\end{abstract}

Keywords: pointing, CARMA, arrays, interferometry, millimeter-wave astronomy

\section{INTRODUCTION}

Optical telescopes are often used to determine initial pointing models for (sub-)millimeter antennas.* The telescope is usually mounted to the backing structure of the antenna and looks through a hole in the antenna surface. The typical approach is to observe a set of stars all over the sky and determine the differences between the predicted, via the encoder and initial pointing corrections, and actual positions of the stars. The difference vectors are then used to determine a set of parameters that correct the encoder readings to provide superior pointing, i.e. pointing model. ${ }^{1}$

The benefits of this approach are great. Sources can usually be detected at signal to noise levels of 10 or more in a second even with small aperture telescopes. This provides positions to small fractions of the telescope resolution or seeing limit, which are typically one to a few arcseconds. Two dimensional optical detectors of sufficient quality are inexpensive whereas many radio receiving systems are single element and expensive. The speed implied by fast measurement of the two dimensional pointing offset, coupled with the far greater and more uniform sky density of stars compared to (sub)millimeter bright sources, results in coverage of the entire sky in a few hours or less.

However, this benefit does not come without cost. The pointing models for the optical and radio telescopes may differ given the localized mounting of the telescope within the backing structure of the antenna. Additional corrections are needed when applying the optical pointing model to the radio telescope. Some of these, like the response of each system to gravity, can be characterized and are constant over periods of weeks or more. Others, like the relative collimation of the beams, can change with temperature. In addition, there are other thermal changes to the pointing which may influence the telescope and antenna differently. Therefore, after determining the common parameters of the telescope and antenna pointing, the telescopes are traditionally not used and/or are removed.

Further author information: (Send correspondence to S. Corder: E-mail: scorder@nrao.edu, Phone: +56 978877681

* In what follows, antenna will refer strictly to a radio system while telescope will refer to an optical system. The system includes the reflector/lens, mount, and the radio receiver/optical camera.

Ground-based and Airborne Telescopes III, edited by Larry M. Stepp, Roberto Gilmozzi, Helen J. Hall Proc. of SPIE Vol. 7733, 77333P - C 2010 SPIE · CCC code: 0277-786X/10/\$18 - doi: 10.1117/12.857431 
At the Combined Array for Research in Millimeter-wave Astronomy (CARMA) the telescopes are permanently mounted to the antennas. This allows us to use of the optical systems during radio observations. In this paper we discuss the use of optical pointing to correct the antenna pointing. Optical offset pointing will refer to the use of difference measurements between the telescope and antenna to correct the antenna pointing whereas optical/radio pointing will refer strictly to pointing with the telescope/antenna. In section 2 we give the specific details of pointing at CARMA. In section 3 we show the results of our initial proof of concept study. In section 4 we present the methods used to implement the optical offset pointing during radio observations. In section 5 we offer a few examples of improvements made by using this method. In section 6 we summarize the results and discuss future directions for further improving this method.

\section{CARMA POINTING}

\subsection{Physical Properties}

CARMA is a 23-element, heterogeneous interferometric array, consisting of six $10.4 \mathrm{~m}$ antennas, nine $6.1 \mathrm{~m}$ antennas, and eight $3.5 \mathrm{~m}$ antennas. It operates at frequencies of $27-35 \mathrm{GHz}, 85-116 \mathrm{GHz}$, and $215-270 \mathrm{GHz}$. Further information on CARMA can be found at www.mmarray.org. The work and original implementation discussed here were performed prior to the introduction of the 3.5-m antennas which are not discussed here. For more information on CARMA without the addition of the 3.5-m antennas see Woody et al. ${ }^{2}$ or Corder et al. in the proceedings for SPIE Conference 7737. For current and ongoing work see contributions by Wright, Perez et al., and Mundy in this proceeding.

The telescopes are mounted in the backing structure of the antennas and look through small holes in the primary surface of the antenna. While the manufacture of the telescopes mounted on the 10.4-m antennas and the 6.1-m antennas differ slightly, their specifications are similar. The telescope systems themselves are composed of a $102 \mathrm{~mm}$ refracting telescope, a motorized lens cap and a COHU 4910 Series CCD video camera. Focusing is achieved manually by rotating the camera body in or out along the threads of a C-mount. The combined optical system results in 1.25 arcseconds per pixel and a field of view of approximately 10 by 15 arcminutes. The camera is connected to a frame grabber that captures individual frames at a rate of 15 frames per second. The camera gain is adjusted automatically depending on incident light levels, allowing for function at day- and night-time.

\subsection{Telescope Software Function}

The frame grabber images are immediately converted to the Python image structure. The centroid is calculated as follows. A user defined number of edge pixels are rejected and the position of the brightest remaining pixel is returned. If two nearly equally bright pixels are adjacent in the map, the initial estimate of center position is taken as the mean location of these two pixels. The rms of the remaining image is taken and pixels below 3 times this level are masked. A subregion of 15 by 15 pixels around the peak is defined and pixels outside this region are also masked. The final returned centroid is the intensity weighted mean position of the unmasked pixels. The correction is applied to the telescope pointing if certain validity conditions are met and the corrections remain in the system if certain convergence criteria are met. The implementation varies from day-time to night-time and is outlined below. Many of the parameters are tunable but the defaults described are well-behaved.

At night, the centroid from 16 frames is calculated. If fewer than half of these are valid, i.e., no valid centroid, then the centering attempt is abandoned. Otherwise, if the formal uncertainty in the centroid position is less than 1.5 arcseconds per axis, the offset is applied to the active antenna pointing model. Uncertainties in the positions are typically $0.2-0.4$ arcseconds per axis. This process is repeated up to four times until the corrections are less than 10 arcseconds. Repeated applications are sometimes needed due to errors in the rotation angle of the camera and the plate scale. Usually for very large initial offsets no more than two rounds are needed. For offsets in typical optical offset pointing runs, one correction is sufficient. If the process does not converge in four attempts, the offsets are removed from the pointing model. By day, the process is more complicated because of the need to do background subtraction. Typically 40 frames are coadded and a single centroid is calculated. Background subtraction is done by nodding the telescope 2 arcminutes off the nominal stellar position and integrating 40 frames. Convergence is checked on the on-source, background-subtracted, coadded frames similarly to the night-time method. A single background frame is used for each of the four possible cycles.

Proc. of SPIE Vol. 7733 77333P-2 


\subsection{Pointing Model and Performance}

The pointing for both 6.1- and 10.4-m antennas can be explained as a basic model with extensions. The basic parameters include the seven fundamental parameters described in Chapter 5 of Baars. ${ }^{1}$ In summary these are a constant (on the sky) collimation term for the azimuth and elevation offsets, an encoder offset for azimuth ${ }^{\dagger}$, a non-perpendicularity of the azimuth and elevation axis, a gravitational sag term, and two terms describing the rotation of the coordinate system due to the tilt of the azimuth axis. The 10.4-m antennas have a system which actively corrects for the tilt of the antenna while the $6.1-\mathrm{m}$ antennas do not. The collimation terms and the gravitational sag are allowed to vary from telescope to antenna. The 6.1-m antennas have additional terms in the model. These account for the asymmetry induced in the yoke due to the non-central location of the cabin and the ellipticity of the azimuth ring. A complete functional form listing of the two pointing models can be found in Chapter 4 of Corder. ${ }^{3}$ For a discussion of additional terms that can in principle be added for physical reasons, see, for example, Mangum. ${ }^{4}$ For the purposes of the discussion here, the telescope and antenna pointing are assumed to have differences in the two beam collimation terms and the response to gravity term.

The details of the radio pointing process at CARMA are contained in White \& Corder. ${ }^{5}$ The basic approach is to make cross patterns of 5 or 9 points per axis, first moving one type of antenna with the second type fixed then reversing the process. The resulting one dimensional voltage patterns are fit by Gaussian profiles to obtain the two dimensional offset. Typical pointing performance at CARMA for telescopes and antennas for various conditions is summarized in Table 1. The limiting magnitudes for the telescopes on both types of antennas are 5 $\mathrm{V}_{\text {mag }}$ and $3 \mathrm{~V}_{\text {mag }}$ for night- and day-time function, respectively. The day-time limit puts a rather serious limit on the day-time utility of any optical offset pointing procedure given the change in limit results in a doubling of the average separation between radio and optical sources in our optical catalog.

\section{PROOF OF CONCEPT TESTING}

\subsection{Equation of Merit}

Productive use of the telescopes to correct antenna pointing requires either the density of optical pointing sources to be much higher than that of radio pointing sources or the time taken to do optical pointing to be much less than time taken to do radio pointing. The time taken to point is relevant when pointing must be done often, e.g., during periods of thermal stress. If the speed of radio pointing was the same as optical pointing and the proximity to pointing sources for the two was the same, there would be clearly no need to use the optical telescopes. Optical pointing is far faster at CARMA, by factors of a few to more than an order of magnitude depending on conditions, and the density of pointing stars is far greater than pointing quasars.

The use of telescopes to correct antenna pointing requires two specific senarios to be addressed. First, the scatter in the difference between the optical and radio pointing offsets must be smaller than the scatter in the radio pointing offsets alone. Second, the difference between these offsets must be constant in time, across the sky and ideally constant over time periods of large thermal stress. Strictly, the scatter in the offsets is subject to a slightly more strict bound, the details of which can be found in Corder, ${ }^{3}$ but the basic equation for the limiting scatter in radio to optical offset vector $\left(\delta R_{v e c}\right)$ is given by

$$
\left(\delta R_{v e c}\right)<|d F / d \phi| \sqrt{|d \phi / d t|\left(\delta T_{r a d}^{2}-\delta T_{o p t}^{2}\right)+C^{2}\left(D_{r a d}^{2}-D_{o p t}^{2}\right)},
$$

where $|d F / d \phi|$ is the magnitude of the pointing error gradient, $|d \phi / d t|$ is the coordinate speed of the source in question, $\delta T_{\text {rad/opt }}$ is the time between the radio or optical pointing measurements (typically several hours and 10-30 minutes, respectively, at CARMA), $C$ is a correction factor to scale on the sky separations to coordinate separations, and $D_{\text {rad/opt }}$ is the distance from science target to radio or optical pointing source (typically more than 20 degrees and less than 10 degrees, respectively, at CARMA). The limiting behaviors are as expected. As the discrepancy between the optical and radio values for time between pointing and distance to pointing source grows small, the error allowance in the radio-optical offset pointing vector becomes small, tending to zero. If the value under the square root becomes negative, no amount of error can satisfy the relation.

\footnotetext{
${ }^{\dagger}$ An elevation encoder offset is degenerate with elevation collimation errors.
} 
Table 1. CARMA Pointing Parameters

\begin{tabular}{|c|c|c|}
\hline Parameter & $10-\mathrm{m}$ & $6-\mathrm{m}$ \\
\hline Short-term Repeatibility ${ }^{\mathrm{a}}$ & $0.5 "$ & $0.5-0.75 "$ \\
\hline Short-term Repeatibility (Radio) ${ }^{\mathrm{a}}$ & $1.5 "$ & $2.5 "$ \\
\hline Post model-fit $\mathrm{rms}$ & $2.1 "$ & $2.0 "$ \\
\hline Blind pointing ${ }^{\mathrm{b}}$ & $2.7 "$ & $3.5-5 ”$ \\
\hline Decay Time ${ }^{\mathrm{c}}$ & $\sim 30$ days & $\sim 14$ days \\
\hline Half-fidelity Limit ${ }^{\mathrm{d}}$ & $3.5 "$ & $6.5 "$ \\
\hline Daytime rms (optical) & $5 "$ & $11 "$ \\
\hline Radio Pointing $\mathrm{rms}$ & $5 "$ & $6 "$ \\
\hline Daytime Radio Pointing $\mathrm{rms}$ & $6 "$ & $11 "$ \\
\hline Limiting $\mathrm{V}_{\text {mag }}$ of Cameras (Night) & 5 & 5 \\
\hline Limiting $\mathrm{V}_{\text {mag }}$ of Cameras (Day) & $<3$ & $<3$ \\
\hline
\end{tabular}

\footnotetext{
a The short-term repeatibility describes the $r m s$ of the centroid values obtained on datasets taken in rapid succession.

${ }^{\mathrm{b}}$ Based on short-term measurements all over the sky typically on the night following the measurement of pointing model.

${ }^{\mathrm{c}}$ Time for the pointing model to decay by $20 \%$ over the blind value.

${ }^{\mathrm{d}}$ Pointing error at which the fidelity of mosaics is reduced by a factor of $\sim 2$, see Corder, ${ }^{3}$ Chapter 2 . This value is valid for the $3-\mathrm{mm}$ band and should be considered to be a factor of two smaller for high-fidelity, 1-mm band observations.
}

Note. - All values are representative of typical, night-time behavior unless otherwise noted. Radio pointing measurements utilize the CARMA software utilities multiMap (S. Corder) for data collection and refPoint (S. White) for calculation of offsets. Day-time camera use involves the coaddition of frames and subtraction of the background. The techniques and proof of concept were by Corder \& Carpenter while the final implementation of the coaddition code was by Beard \& Scott. The V-band limits are approximate. Day-time optical limits depend strongly on the angular distance between the source in question and the sun.

The advantage of using optical offset pointing is determined by the ratio of the right to left side of expression 1 . A value of 3-4 implies a large gain while a value of 1.1 or less implies relatively modest gain. We have already discussed why optical pointing is much faster than radio pointing. Then, for a given optical brightness limit and radio pointing source flux limit, which to a large degree determine the distance between target and pointing source, the magnitude of this ratio depends most strongly on the size of error gradients. These error gradients depend on, among other things, the magnitude of thermal stresses. The brightness limits in the radio are determined by the sensitivity of the array in a given wavelength band. The optical sensitivity is limited by the aperture of the optical telescope, the type of receiving system and the sky brightness, i.e. distance to the sun. At CARMA, the optical catalog has been specifically designed to provide a night-time detectable optical pointing source within approximately 7.5 degrees of any location on the sky. For 2 hours per radio pointing, 20 minutes per optical pointing, $\mathrm{C} \sim 1$, error gradient ranging from 0.06-0.9 arcseconds per degree depending on the quality of the pointing model and the amount of thermal stress, and coordinate speeds ranging from 15 to 50 arcseconds per second, the allowed scatter can be anywhere from 2.5 to over 100 arcseconds rms. The allowed error then depends sensitively on these errors in the pointing model and the source elevation.

\subsection{Procedure}

We conducted three sets of tests to verify that the difference between antenna and telescope pointing is constant in time, across the sky, and during periods of thermal stress. The first two assumptions are inherent to the CARMA pointing model and we are simply verifying them. The third test requires that we measure the optical to radio offset vector at night-time and compare the results to day-time and sunrise/sunset behavior.

Test data was collected as follows. Software to find optical counterparts for radio targets was used offline to 
select antenna pointing sources that had optical counterparts, detectable by the CARMA telescopes, within 10 degrees. Typical separations were 5 degrees by night and 10 degrees by day. I will refer to the combinations of telescope and antenna pointing sources as pointing pairs.

Stable night-time conditions were used as the baseline measurement for the differences between telescope and antenna pointing. Pointing pairs were measured alternating between telescope and antenna pointing as quickly as possible. Pairs were measured over the entire sky and the list of pairs was repeated several times. A similar test was made following the same target over the course of several hours. The same tests were repeated during day-time. One additional test was to measure the pointing difference over sunrise, the period which should result in the most thermal stress to the antenna mount.

Each pointing pair cycle took about 8-10 minutes, most of which was attributed to the radio pointing time. Night-time observations of the repeated source test were taken 10 July 2006 and 02 November 2007 and contained 23 and 50 pairs, respectively. The all-sky night-time data was taken on 29 March 2007 and contained 30 points. Day-time data over the entire sky was taken on 01-02 November 2007 (23 pairs) and 22-23 November 2007 (50 pairs). Repeated source measurements were taken only once, 29 November 2007 and contained only 10 points. Thermal stress tests were performed at various times with the specific example of data from 10 July 2006 shown below. This consisted of measurements of 3c454.3 (flux $\sim 10 \mathrm{Jy}$ ) and HP113963 ( $\mathrm{V}_{\text {mag }}=2.5$ ) from 9 to $15 \mathrm{UT}$ with sunrise occurring at 12:48 UT.

\subsection{Test Results}

Table 2 provides the details of the rms estimates for radio and radio-optical offsets for night- and day-time use. If the $\delta R_{v e c}$ values have greater $r m s$ than the radio pointing measurements, then optical offset pointing certainly does not help the situation. Often there are systematic trends in the pointing offsets but a comparison of the rms of these trends still results in the quantity with the smaller trend also having the smaller rms. Typically for the 10.4-m antennas, the repeated source observations and measurements all over the sky and on repeated sources at night have $r m s$ of about 3.5-5". The 6.1-m antennas have slightly larger $r m s$ values of 4.5-5.5". During the day, these numbers increase. For the 10.4-m antennas the azimuth scatter grows significantly and is on average larger than the radio pointing alone value. However, this is not the case in elevation, where the elevation scatter is often much smaller than the radio value. The combined $r m s$ is between 5.5 and $7.5 "$. In the case of day-time observations with the $6.1-\mathrm{m}$ antennas, the optical to radio offset $r m s$ is almost always significantly smaller than the radio values alone, indicating that there are systematic trends in the radio pointing. The rms of the vector components is comparable in both axes and is about 7".

If these values are compared to the allowed, night-time values given by expression 1 , it is found that for a well-behaved mount model, i.e. mount error derivatives of the order 0.06 " per degree, the use of optical offset pointing is actually detrimental to the pointing for sources at low elevation, if the radio pointing source is at or within $25^{\circ}$ and pointing is done every two hours at night. This is because the additional scatter in the optical to radio offset vector is greater than the systematic error introduced by the evolution of the pointing model. This is the typical situation for the 10.4-m antennas. The 6.1-m antennas often have error gradients more on the order of 0.1 to 0.2 . In these cases, it is beneficial to use optical offset pointing.

By day, an odd result emerges. It appears as though the 10.4-m antennas would benefit from optical offset pointing in elevation but not in azimuth. If both axes are corrected, the degree of improvement or detriment depends on the magnitude of the elevation error gradient. A more detailed exploration of the systematics is required to address this. For the 6.1-m antennas, it is clearly beneficial to use optical offset pointing in all cases. A benefit not considered here is that the use of optical offset pointing always provides time benefits over radio pointing alone.

Aside from this decrease in pointing rms over typical observing conditions, systematic trends are also removed. Figure 1 shows the change in elevation offset as a function of time over a period of thermal stress, i.e. sunrise. One 10.4-m antenna, seen in the left panel, has a systematic change associated with sunrise. Here the pointing changes by $\sim 12 "$ over the course of two hours. Given the width of the primary beam at $\lambda=1.3 \mathrm{~mm}$ is about 30 ", an observation would have almost had the half power point of the beam pointed at the source and the sensitivity would be reduced by nearly a factor of two for this antenna. The damage to mosaic images would have been 


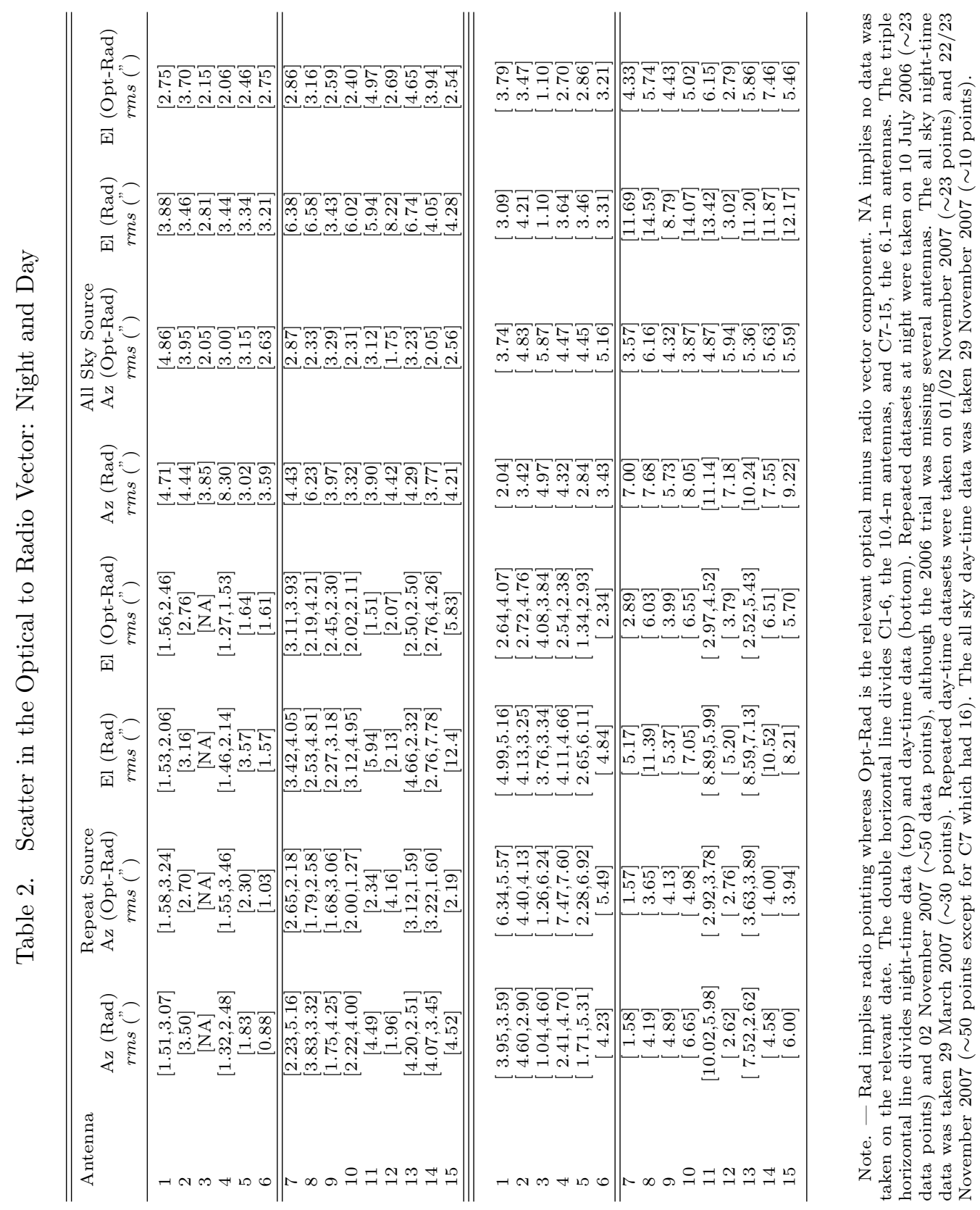

Proc. of SPIE Vol. 7733 77333P-6 
far greater. These periods of thermal stress are times when the magnitude of the gradient in the elevation error field is large enough that the introduction of increased $r m s$ in azimuth would be more than counteracted by the correction of this large elevation change. In the right panel, C13 is experiencing both a systematic change due to thermal stress and an error in the pointing model. Here the pointing changes by over 30 " over the course of just 3 hours and 30 minutes. In both cases the pointing is evolving at 6-9" per hour. Radio pointing every hour is both inefficient and insufficient in such situations. Corrections every 20-30 minutes would allow sufficient time resolution for the correction. Optical offset pointing allows this.
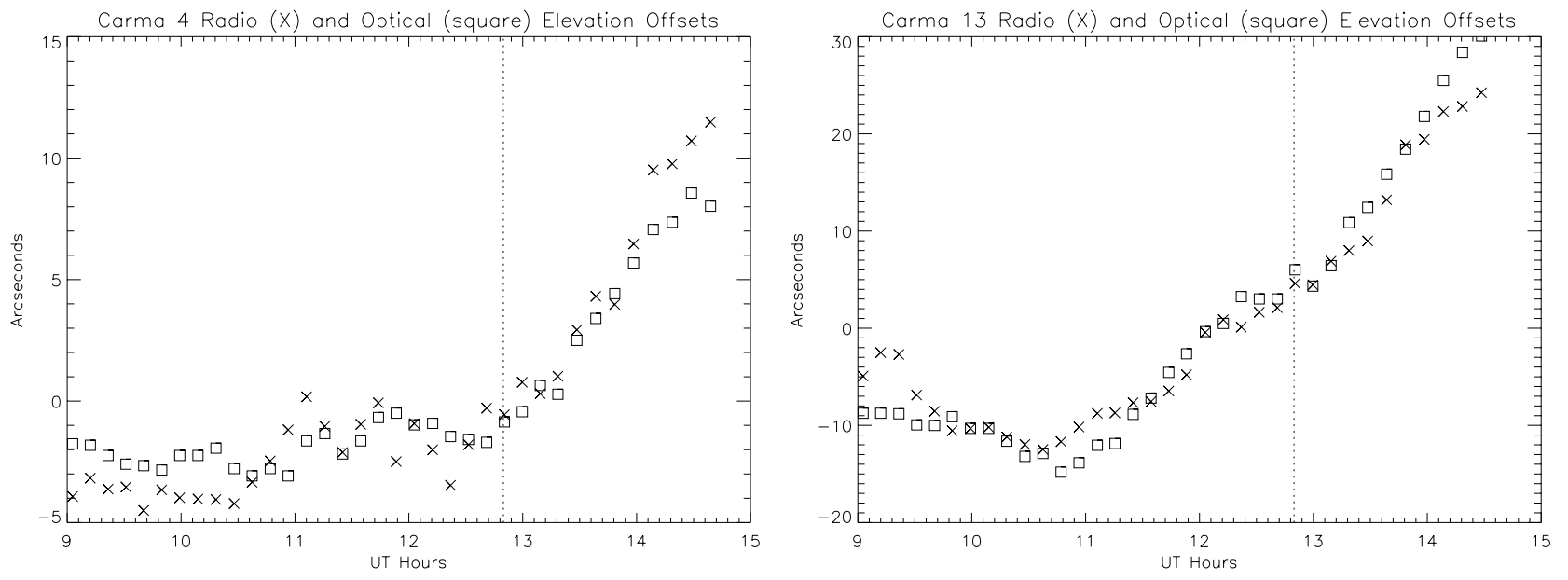

Figure 1 Optical and radio elevation offsets are plotted versus time. The $\mathrm{X}$ points are radio offsets while the squares are optical offsets. The left panel is the pointing offset of $\mathrm{C} 4$ under the thermal stress of sunrise, denoted by the dashed line. The right panel displays the elevation offsets of $\mathrm{C} 13$ over the same time period.

In general, systematic trends persist in the optical to radio offset vector. The magnitude of these systematics is typically reduced for the $6.1-\mathrm{m}$ antennas with the use of optical offset pointing. There are a few instances at night where the trends are somewhat larger in the optical to radio offset but in general the systematics are reduced. The case for the 10.4-m antennas is somewhat less clear. At night, the systematic evolution in the radio elevation term is almost always greater than the trend in elevation for the radio to optical offset. However, there is not such clear evidence for the evolution of the azimuth components. The evolution of the radio pointing alone in the azimuth component is typically smaller or comparable in magnitude to the evolution of the optical to radio offset, again arguing that perhaps the optical offset should only be applied to elevation. By day, the result is similar. The elevation component of the optical to radio offset typically has less systematic variation than the radio pointing alone but this is clearly not the case azimuth. The entire discussion of whether the azimuth component of optical offset pointing should not be applied is complicated by the fact that observations sometimes show systematic trends and obvious results but on subsequent attempts the trend is absent. This is likely an example of the hysteretic effect of thermal heating or wind on pointing.

\subsection{Summary}

The method seems to work well in concept. If there are serious problems with the pointing model, the method corrects for them. The application of the offsets to the 10.4-m antennas will result in comparable pointing at night and comparable or slightly improved pointing by day. Possible small detriments to some of the 10.4-m antenna pointing $r m s$ values are offset by correction of possible large errors in other 10.4-m antennas. For the 6.1-m antennas, the picture is far clearer. The pointing performance is almost always improved by optical offset pointing.

The reduced time to measure the pointing results in increased time observing and sensitivity for the antenna. A key benefit is gain stability in the outer portions of the primary beam, which is susceptible to small errors in pointing. See Chapter 2 of Corder ${ }^{3}$ or Cornwell et al. ${ }^{6}$ for a review of the impacts of pointing offset on image 
quality. The method provides great advantages in both the on-source time and image quality because the benefit is always large, both in terms of day-time and night-time operation. So we have integrated optical offset pointing as a regular observing procedure.

\section{IMPLEMENTATION}

In regular operations we have implemented a system that allows the integration of measurements made with the telescopes with standard radio observations. If the user indicates that they would like to use the telescopes to update their pointing the following sequence of observations is excited. In the remainder of this section, user definable parameters are written as parameterName (default value). For online documentation see www.mmarray.org under "Observing with CARMA" and the FAQ under "Script Writer and Instructions" .

The basic approach is to have n-many copies of the control system running where $\mathrm{n}$ is the number of antennas. One of these controls the main functions of the array and serves as a master while the other n- 1 act as watchers most of the time. Upon completion of a specific radio observation, the master window can initiate a signal that causes the other n- 1 control sessions to take control of an individual antenna and slew it to the telescope pointing source. The master session also takes an antenna. Upon completion of their telescope pointing session, the watcher sessions send a signal indicating that they have completed their execution. When all watchers have sent that signal and the master has also completed its telescope pointing, the master session assumes control of the entire array of antennas again and proceeds with additional radio observations.

During the course of a full round of standard radio science observation the following steps are taken. First an attempt to measure the pointing differences between the telescopes and the antennas is made. This is done by first doing a antenna pointing on a bright radio source. An online program then automatically selects the most nearby appropriate counterpart which is then pointed on optically. The appropriateness is based on whether or not the sun is up and the magnitude limit is changed accordingly and is also under some user control. The value of maxmag (None, defaults $\mathrm{V} \sim 3$ by day and $\mathrm{V} \sim 5$ by night) determines how faint the optical counterparts are allowed to be. Also, maxOptSep (20) controls how far away the user is willing allow their telescope counterpart to be from their antenna target. The typical separations are 7.5 degrees by night and 15 degrees by day with considerable scatter by day. The user is also allowed to select a minimum (optradElmin (30)) and maximum (optradElmax (75)) elevation at which to measure the pointing difference. The user can alternatively select a preferred source with optradPreferred (None) and that source is used if it is up. Several sources can be specified.

A difference in the pointing directions is calculated. If the number of failed antennas is greater than nbadAnts (3) then telescope based pointing is aborted until the next scheduled antenna pointing according to intervalDay ( 2 hours), intervalNight (4 hours) or intervalTran (not set, 2 hours after sunrise/sunset), depending on which is appropriate. If the attempt to measure the telescope and antenna pointing difference is considered successful, the values are stored for the course of the current radio observation and will be referred to as the pointing difference vector.

If successfully measured, this pointing difference vector is assumed constant over the course of the radio observation. From then on pointing updates are done via the telescope. Telescope pointing updates are combined with the pointing difference to correct the antenna pointing. The user specifies intervalOpt (0.5 hours) to specify how often optical pointing updates should be done. Pointing on flux calibration and passband calibration sources is only done if the user has specified these be done for the radio pointing case (doPoint in the passband and flux parameters areas). Once the standard phase calibrator/science target cycle is started, the parameters optPointCal (True) and optPointTarget (False) determine if the telescope pointing source is selected to be close to the science target or the phase calibrator. If the telescope pointing fails according to nbadAnts for nfailOpt (3) rounds of pointing, then optical pointing is aborted and the parameters of antenna pointing are reinstated. This can happen if weather conditions change appreciably over the course of the observation.

Additional options exist that control how the stellar imaging is done by the telescope. These include background subtraction via an antenna nod with subtract (None, i.e., do it by day but not at night), control over the number of centroiding attempts via centroidLoopMax (16), and control over the number of images to coadd via ncoadd (None, which is 40 by day and 1 by night). The user also has additional control over the ability to 
tune to a 3-mm band frequency for better radio pointing (optradTune95 (False)) which is useful for 1-mm band observations in some cases.

There is a substantial gain in speed for this method over traditional antenna pointing at CARMA. There are also cases where the pointing can be considerably improved. The failure modes were designed to handle the case of clouds well. If the user is really concerned about clouds being a problem, the value of nfailOpt can be made smaller so that if their are patchy clouds in the direction of their target but not the pointing difference source, no significant time is wasted. In practice, the time lost during night-time observations is small, given the speed of the process and lack of a need to doing background subtraction. However, the need for the method by day is much larger. The default mode for pointing at CARMA is to doOptPoint True.

\section{IMPROVEMENTS}

The best evidence to outline the success or failure of the method is not to discuss rms values for various pointing constants but to apply the method to real observations and see what happens. Figures 2 through 4 display that evidence. Figure 2 shows two different observations taken 48 hours apart using the same interferometric gain calibrator. The weather conditions were nearly identical for these observations. The left panel shows an observation at $90 \mathrm{GHz}$ without the use of optical offset pointing. This observation, taken at night, followed the standard guideline of pointing every four hours. Clearly systematic trends are visible in the data. The right panel describes a dataset taken at $115 \mathrm{GHz}$ with optical offset pointing. The total offset applied by the optical pointing was about 0.4 arcminutes and easily accounts for the systematic trend seen in the previous days data without optical offset pointing. The increased scatter in the amplitudes of the right panel over the left panel can be almost entirely explained by the increased noise at this frequency.

Figures 3 and 4 describe a $230 \mathrm{GHz}$, day-time observation. Figure 3 shows the corrections applied in both azimuth (left) and elevation (right) to two representative antennas, one 10.4-m (top) and one 6.1-m antenna (bottom). The 10.4-m antenna shows changes of 8 and 10 arcseconds per axis or 12 arcseconds total. Such a shift on the 10.4-m baseline would result in a decrease of nearly a factor of two in sensitivity if combined with other, similar 10.4-m antennas. The offsets in the 6.1-m antenna case are more dramatic. Without the applications of these offsets, the 6.1-m antenna would have had the targeted pointed well outside the main beam. However, Figure 4 shows the amplitude from two baselines using this data. The variation is $20 \%$ over the course of the observation and most of the systematic trend is removed. Clearly optical offset pointing allowed a successful observation when otherwise more than half of the baselines would have had their target positioned at below the half power point in the beam. Even following the radio pointing proscription of pointing once every two hours, the trends in pointing were so large that significant sensitivity and image quality would have been lost.

While not an excellent diagnostic of the applicability of optical offset pointing, the ratio between the rms of radio pointing terms to the rms of the optical to radio offset vector is something that has been tracked long-term at CARMA. Table 3 shows the radio pointing $r m s$ and the ratio of radio pointing $r m s$ to the optical radio offset rms for all the antennas during day-time, night-time, sunrise and sunset. While there are two 10.4-m antennas that have ratios of less than 1 , indicating that there is more scatter in the optical to radio offset, the general trend is impressive with all 6.1-m antennas showing marked improvement at all times. Most 10.4-m antennas show improvement during the most stressful times for pointing and only one antenna shows clear detriment and this is only over sunset.

\section{SUMMARY \& FUTURE}

The speed, pointing improvement and ease of use have made optical offset pointing the default pointing mode at CARMA. The support software has been configured such that the user need only flip a single boolean flag to turn the method on and off. All of the complication of counterpart selection is done internally and adjusted given the ambient conditions. Failure modes are reasonable, making the transition from traditional radio pointing to optical offset pointing efficient and smooth. Time benefits of the method over radio pointing range from factors of several to more than an order of magnitude meaning that pointing can be performed 3-4 times as often with often considerable time savings. The examples of data collected using this method show substantial gains in

Proc. of SPIE Vol. $773377333 P-9$ 

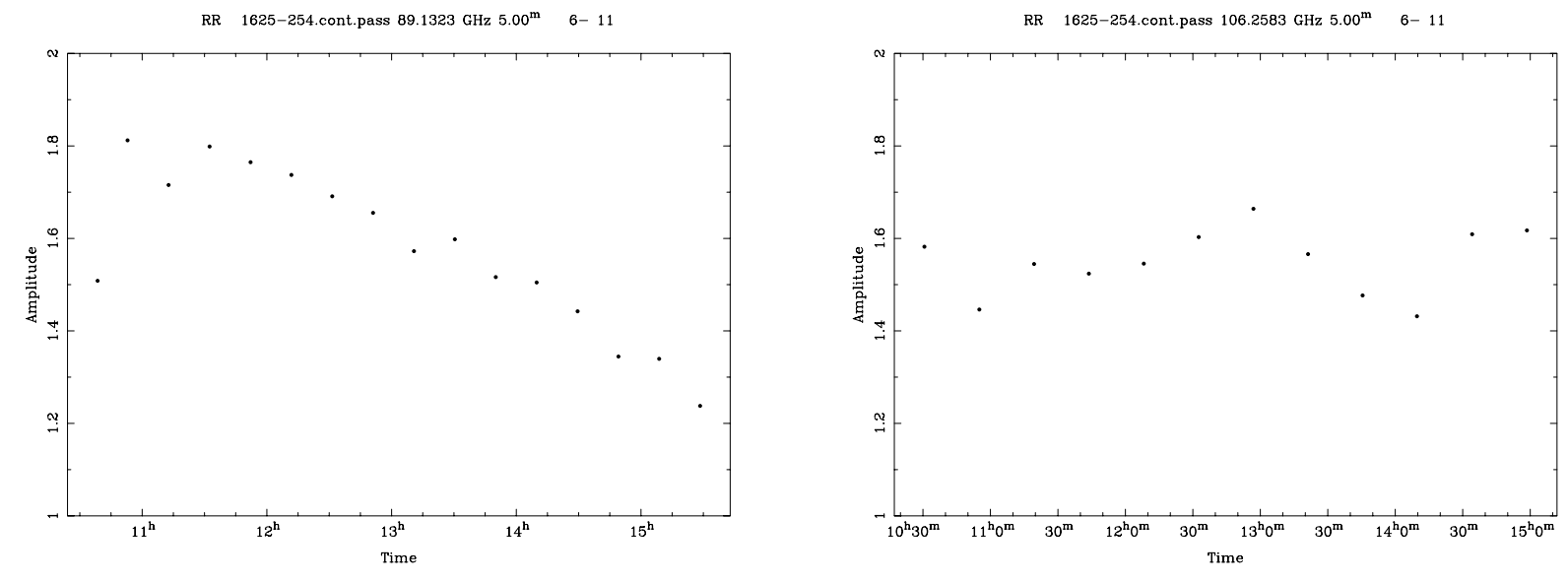

Figure 2 The left and right panels show amplitude (in Jy) versus time for a specific baseline of antenna 11, an antenna whose mount model was corrupted by environmental effects since the last derivation of a new pointing model. The source is the same and the datasets are separated by two days. The left panel is a $90 \mathrm{GHz}$ observation by Lommen et al. without optical offset pointing. The right panel is a $110 \mathrm{GHz}$ observation by Andrews et al. with optical offset pointing.

Table 3. Three Week Average rms of Radio Offsets vs. Optical-Radio Offsets

\begin{tabular}{|c|c|c|c|c|c|c|c|c|}
\hline Antenna & $\begin{array}{c}\text { Sunset } \\
r m s \text { pointing } \\
\text { (") }\end{array}$ & Ratio & $\begin{array}{l}\text { Night } \\
r m s \text { pointing } \\
\text { (") }\end{array}$ & Ratio & $\begin{array}{c}\text { Sunrise } \\
r m s \text { pointing } \\
\text { (") }\end{array}$ & Ratio & $\begin{array}{c}\text { Day } \\
r m s \text { pointing } \\
\text { (") }\end{array}$ & Ratio \\
\hline 1 & 10.6 & 1.47 & 11.0 & 1.37 & 10.2 & 1.04 & 11.1 & 1.44 \\
\hline 2 & 6.2 & 0.82 & 5.2 & 0.80 & 6.4 & 1.15 & 7.6 & 1.20 \\
\hline 3 & 8.3 & 1.15 & 8.2 & 1.28 & 8.7 & 1.13 & 9.8 & 1.18 \\
\hline 4 & 8.3 & 0.61 & 7.2 & 0.85 & 7.8 & 0.91 & 9.0 & 0.92 \\
\hline 5 & 6.0 & 1.24 & 4.3 & 1.23 & 6.5 & 1.17 & 6.8 & 1.15 \\
\hline 6 & 9.7 & 1.08 & 9.4 & 1.34 & 11.8 & 1.15 & 11.8 & 1.38 \\
\hline 7 & 8.9 & 1.31 & 8.1 & 1.56 & 99.4 & 1.30 & 14.9 & 1.42 \\
\hline 8 & 9.3 & 1.38 & 8.3 & 1.64 & 10.0 & 1.46 & 11.3 & 2.62 \\
\hline 9 & 10.3 & 1.48 & 8.9 & 1.40 & 9.2 & 1.26 & 14.0 & 1.74 \\
\hline 10 & 10.6 & 1.47 & 8.7 & 1.13 & 8.9 & 1.07 & 15.7 & 1.68 \\
\hline 11 & 11.5 & 1.93 & 6.8 & 1.60 & 9.2 & 1.31 & 13.8 & 2.68 \\
\hline 12 & 11.0 & 1.58 & 8.3 & 1.28 & 9.8 & 1.05 & 13.2 & 1.55 \\
\hline 13 & 11.5 & 1.40 & 7.2 & 0.96 & 8.5 & 1.09 & 13.2 & 1.49 \\
\hline 14 & 9.0 & 1.63 & 10.3 & 1.71 & 11.3 & 1.56 & 16.8 & 2.64 \\
\hline 15 & 7.7 & 1.20 & 6.8 & 1.46 & 7.8 & 1.41 & 14.9 & 2.48 \\
\hline
\end{tabular}

Note. - Data is taken over the last three weeks. The $r m s$ pointing is the $r m s$ in both axes combined for radio pointing and the ratio describes that value over the $\mathrm{rms}$ of both axes combined for the radio-optical vector. The double horizontal line divides the 10.4-m antennas (top) from the 6.1-m antennas (bottom).

amplitude stability suggesting the more frequent update of pointing terms results in increased sensitivity and image quality.

There are several experiments that may allow the method to be further enhanced. The day-time optical pointing model has never been properly characterized at CARMA. Historically this was due to the lack of methods to optically point when the sun was up. However, now, with these methods in hand, it should be relatively straight-forward to determine what, if any modification to the entire pointing model, radio or optical, is needed for day-time function. Cheap, off-the-shelf CCD cameras could be employed to give deeper optical imaging, 

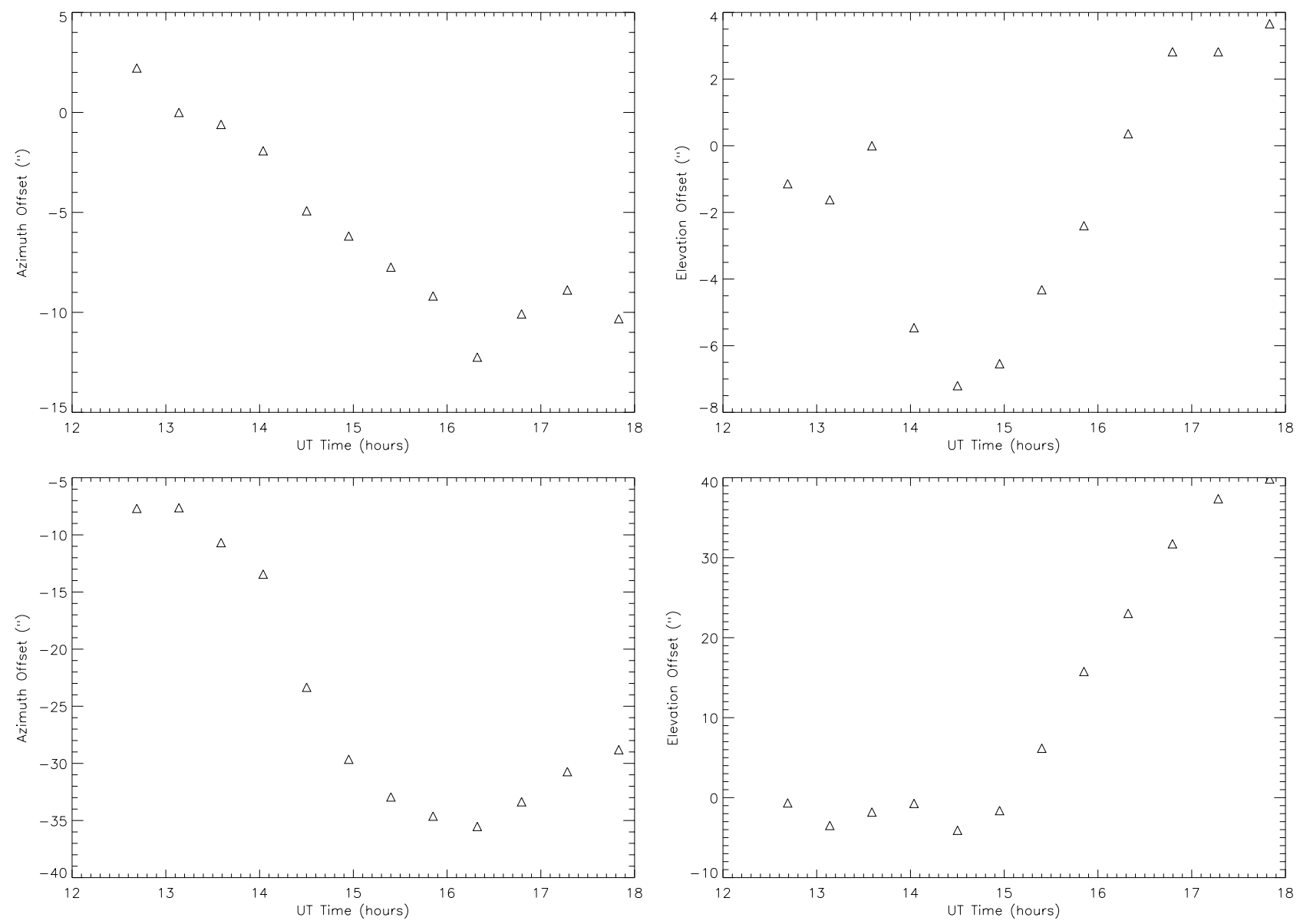

Figure 3 The offsets applied by the online optical pointing correction program are plotted in arcminutes against UT time. The top row shows the offsets for antenna 1, although the magnitude of the change and the functional behavior is similar to other 10.4-m antennas. The left side shows the evolution of the azimuth offset. The right side shows the evolution of the elevation offset. The bottom row shows similar plots for antenna 13. Again, the magnitude and functional form of the changes are typical among 6.1-m antennas.

possibly improving the function of the method, especially by day when a few visual magnitudes improvement in the telescope function could result in substantial gains to the pointing accuracy. If the camera performance were sufficiently good, one could consider active guiding of radio observations with the optical systems. However, significant complication comes with attempting this improvement. We may also improve the overall optical offset pointing performance by including a flexure term to the optical pointing model. The inclusion of a temperature dependent term could also yield a few positive results for the performance of the overall method.

The method does have a few failings. Substantial improvement to the speed and sensitivity of radio pointing at CARMA would mean that optical offset pointing during the night-time would become obsolete. The substantial increase in sensitivity is happening due to the increase in correlator bandwidth currently ongoing at CARMA. If this gain is coupled with increased speed of the radio pointing routine and barring improvements to the optical camera system, night-time, 3-mm band radio pointing will become the preferred method. However, even in that case, 1-mm band and day-time observations will still benefit from the optical offset pointing method. 

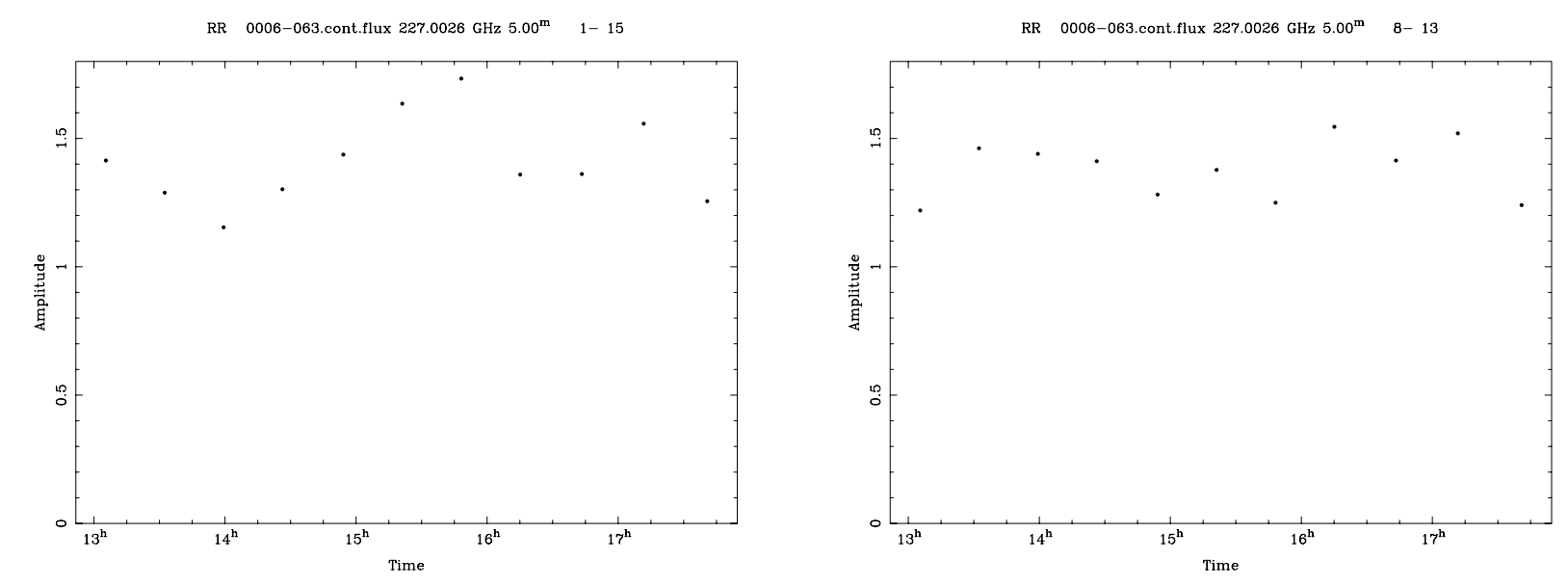

Figure 4 Two baselines of the Maness et al. dataset are shown. The left panel shows the amplitudes of 0006-063 plotted versus time for a baseline antennas 1 and 15. The right panel shows a similar plot for antenna 8. Other antennas show comparable stability.

\section{ACKNOWLEDGMENTS}

The authors would like to thank Andy Beard, Steve Scott and Peter Teuben for working to create and optimize some of the lower level code utilized in this work. Support for CARMA construction was derived from the states of California, Illinois, and Maryland, the James S. McDonnell Foundation, the Gordon and Betty Moore Foundation, the Kenneth T. and Eileen L. Norris Foundation, the University of Chicago, the Associates of the California Institute of Technology, and the National Science Foundation. Ongoing CARMA development and operations are supported by the National Science Foundation under a cooperative agreement, and by the CARMA partner universities.

\section{REFERENCES}

[1] Baars, J. W. M., ed., [The Paraboloidal Reflector Antenna in Radio Astronomy and Communication], Astrophysics and Space Science Library 348 (2007).

[2] Woody, D. P., Beasley, A. J., Bolatto, A. D., Carlstrom, J. E., Harris, A., Hawkins, D. W., Lamb, J., Looney, L., Mundy, L. G., Plambeck, R. L., Scott, S., and Wright, M., "CARMA: a new heterogeneous millimeter-wave interferometer," in [Society of Photo-Optical Instrumentation Engineers (SPIE) Conference Series], C. M. Bradford, P. A. R. Ade, J. E. Aguirre, J. J. Bock, M. Dragovan, L. Duband, L. Earle, J. Glenn, H. Matsuhara, B. J. Naylor, H. T. Nguyen, M. Yun, \& J. Zmuidzinas, ed., Society of Photo-Optical Instrumentation Engineers (SPIE) Conference Series 5498, 30-41 (Oct. 2004).

[3] Corder, S. A., Optimizing Image Fidelity with Arrays, PhD thesis, California Institute of Technology, Pasadena, California (September 2008).

[4] Mangum, J. G., "A telescope pointing algorithm for ALMA," Tech. Rep. ALMA Memo 366, Atacama Large Millimeter/Submillimeter Array (2001).

[5] White, S. M. and Corder, S. A., "Radio pointing measurements for CARMA," Tech. Rep. CARMA Memo 40, Combined Array for Research in Millimeter-wave Astronomy (2007).

[6] Cornwell, T. J., Holdaway, M. A., and Uson, J. M., "Radio-interferometric imaging of very large objects: implications for array design," A\&GA 271, 697-+ (Apr. 1993). 